\title{
The RPC hits in the CMS muon reconstruction
}

\author{
Min Suk Kim* On behalf of the CMS Collaboration \\ Sungkyunkwan University \\ Department of Physics, 300, Chunchun-dong, Jangan-gu, Suwon, Korea \\ E-mail: minsuk@cern.ch
}

\begin{abstract}
Muon tracks in the events collected by the Compact Muon Solenoid experiment at the Large Hadron Collider are reconstructed using the Drift Tubes in the central barrel region, Cathode Strip Chambers in the endcap region and Resistive Plate Chambers (RPC) in both the barrel and endcaps. The RPC system is mainly used as trigger detector, however it also contributes to the muon reconstruction. The number of reconstructed hits in the RPC chambers crossed by the muons with the high transverse momentum $\left(p_{T}>20 \mathrm{GeV} / c\right)$ coming from $Z^{0}$ decays can be used to study the efficiency for single muons reconstructed offline. The muon reconstruction efficiencies with and without the inclusion of the RPC measurements in the track fitting are reported here.
\end{abstract}

XI workshop on Resistive Plate Chambers and Related Detectors - RPC2012,

February 5-10, 2012

INFN Laboratori Nazionali di Frascati Italy

\footnotetext{
* Speaker.
} 


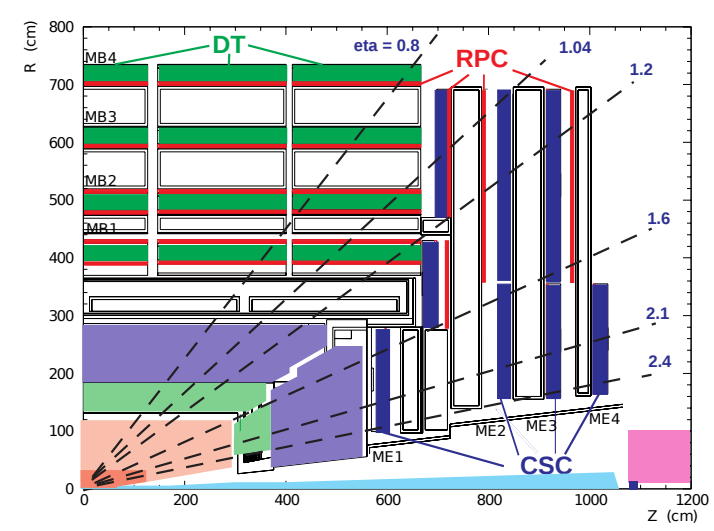

Figure 1: Layout of one quadrant of CMS. The four DT stations in the barrel (MB1-MB4, green), the four CSC stations in the endcap (ME1-ME4, blue), and the RPC stations (red) are shown.

\section{Introduction}

The key to new discoveries and precision measurements at the Large Hadron Collider (LHC) is the ability of excellent muon identification, triggering and precise momentum reconstruction with high efficiency. The muon system of the Compact Muon Solenoid (CMS) experiment [1] and the muon reconstruction algorithms have been designed to achieve these goals. The focus of this report is the contribution of the RPC sub-system to the muon identification and reconstruction capabilities, alongside the Drift Tubes (DT) and Cathode Strip Chambers (CSC) independent sub-systems.

Due to the redundancy provided by the combination of DT/CSC and RPC in the barrel/endcap region, it is expected that the inclusion of the RPC measurements in the track fitting can improve the reconstruction of low momentum muons, especially in the case where the muons escape through the gaps between the wheels in which the chambers are divided along the axis parallel to the beam, leaving hits in only one DT or CSC station. Thus, the analysis presented here focuses on how much the inclusion of the RPC hits will make an impact on the reconstruction of the muon tracks from $Z^{0} \rightarrow \mu^{+} \mu^{-}$with $p_{T}>20 \mathrm{GeV} / c$. To better understand and measure the muon efficiency, the RPC reconstructed hits from all stations when a muon passes through the RPC have been studied.

\section{Detector Layout}

RPCs are used in both the barrel and endcaps as dedicated trigger detectors, covering the range of $|\eta|<1.6$. They complement the muon tracking devices defined by DTs $(|\eta|<1.2)$ in the barrel and CSCs $(0.9<|\eta|<2.4)$ in the endcaps (Figure 1). The use of three different technologies defines three regions in the RPC detector, referred to as barrel $(|\eta|<0.8)$, overlap $(0.8<|\eta|<1.2)$, and endcap $(1.2<|\eta|<1.6)$.

In the barrel region the muon chambers are organised in four coaxial stations, interleaved with iron return yokes. Each of the two inner stations (MB1, MB2) contains a layer of DTs sandwiched between two layers of RPCs, whereas each of the two outer stations (MB3, MB4) consists of one layer of RPCs and one layer of DTs. These barrel stations are grouped into five wheels, which are in turn divided into twelve $\phi$ sectors. The endcap region is composed of three iron disks holding a total of three RPC planes and four CSC planes on each side (plus/minus) of the CMS detector. 

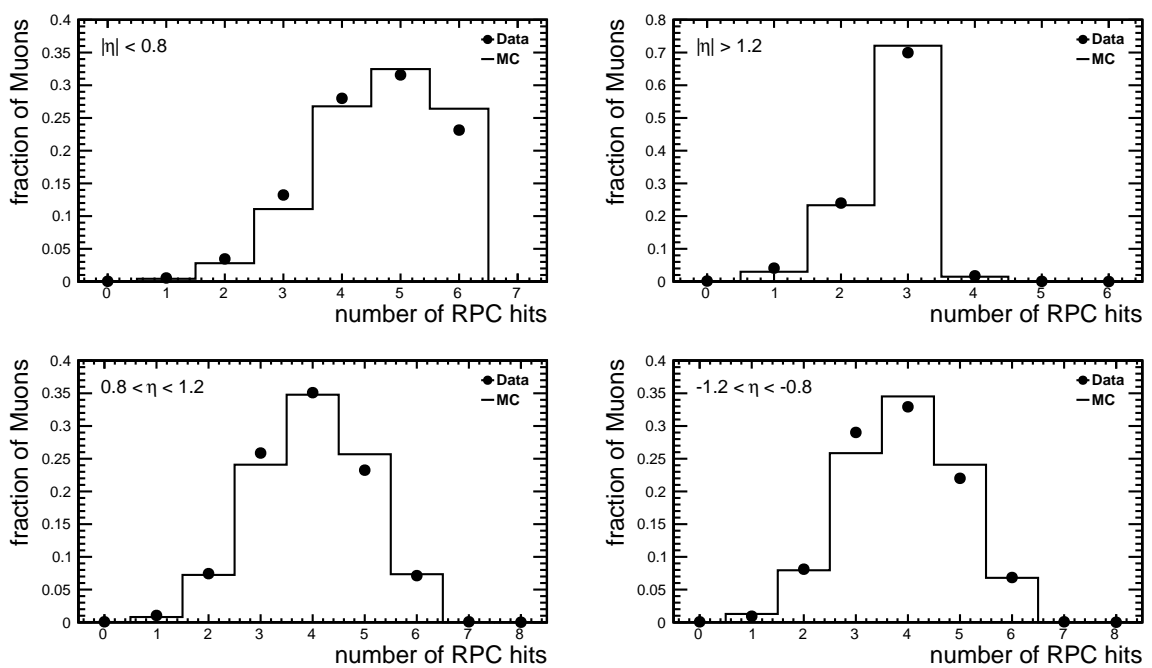

Figure 2: Number of RPC hits per global muon for the barrel, endcap, overlap minus and plus regions (clock-wise, starting from top-left). Results are shown for both data (dots) and Monte Carlo (solid line).

\section{RPC Hits in Muon Reconstruction}

In the standard CMS reconstruction, tracks are first reconstructed independently in the silicon tracker (tracker track) and in the muon system (standalone-muon track). A global-muon track is fitted combining hits from the trackers and standalone muon track [2]. The majority of muons from collisions are reconstructed either as a Global Muon or a Tracker Muon, and very often as both. However, if both approaches fail and only a standalone-muon track is found, the muon is a Standalone Muon.

If at least one muon segment (i.e. a short track stub made of DT or CSC hits) matches the extrapolated track, the corresponding tracker track qualifies as a tracker-muon track. However, for the reconstruction of the standalone- or global-muon track, at least two measurements, one of which must be of the DT or CSC segment, must be matched. Figure 2 shows the number of RPC hits per global muon for the barrel, overlap and endcap regions. Figure 3 shows the average number of RPC hits of global muons as a function of $\eta$ and $\phi$ for given $\eta$ regions. The dips in the barrel $\eta$ around \pm 0.3 and \pm 0.8 are due to the gaps between the wheels. Similarly, the oscillations in the barrel $\phi$ distribution are due to the cracks between the adjacent sectors. There is a small bias from the event selection with at least one muon triggered.

\section{Muon Efficiencies with and without RPC}

In this report we measure the efficiencies of two types of selections with $p_{T}>20 \mathrm{GeV} / c$ based on various muon identification variables: Loose and Tight selections. Both types of selections are the standard CMS selections with the isolation requirements based on the tracker and calorimeters, but the tight muon selection includes additional quality requirements on the muon tracker track.

In order to measure the muon reconstruction efficiencies with and without the inclusion of RPC in the track reconstruction, one can use the events with $Z^{0}$ decays into a pair of muons and consider one of the muons as the tag to probe the efficiency using the other muon. The passing probes 

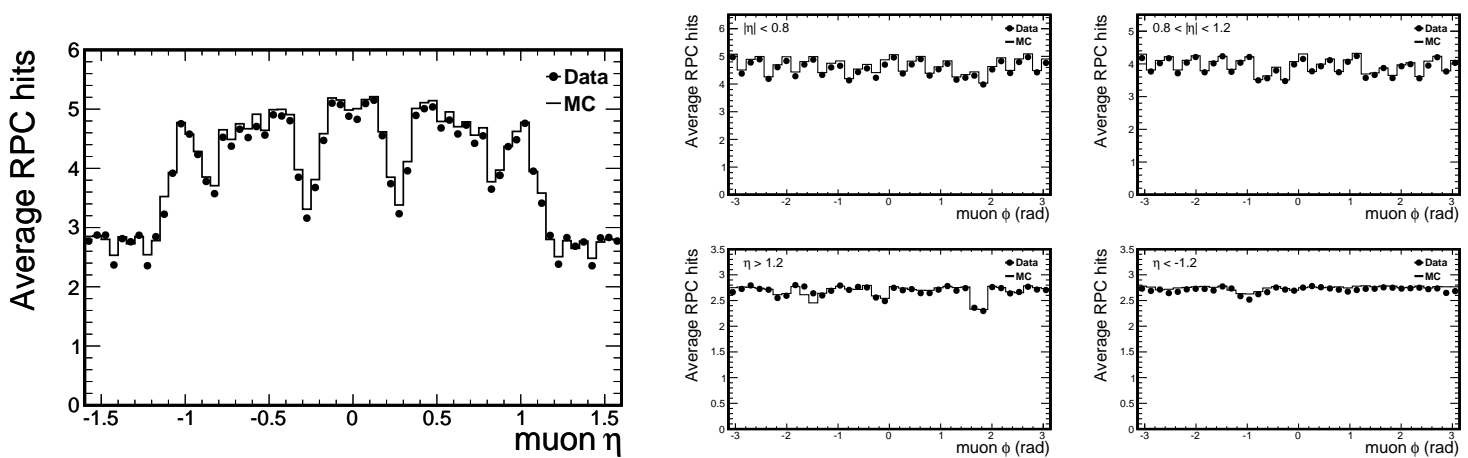

Figure 3: Average number of RPC hits of global muons vs. $\eta$ (left) and $\phi$ for the barrel, overlap, and endcap minus and plus regions (right four plots, clock-wise, starting from top-left).
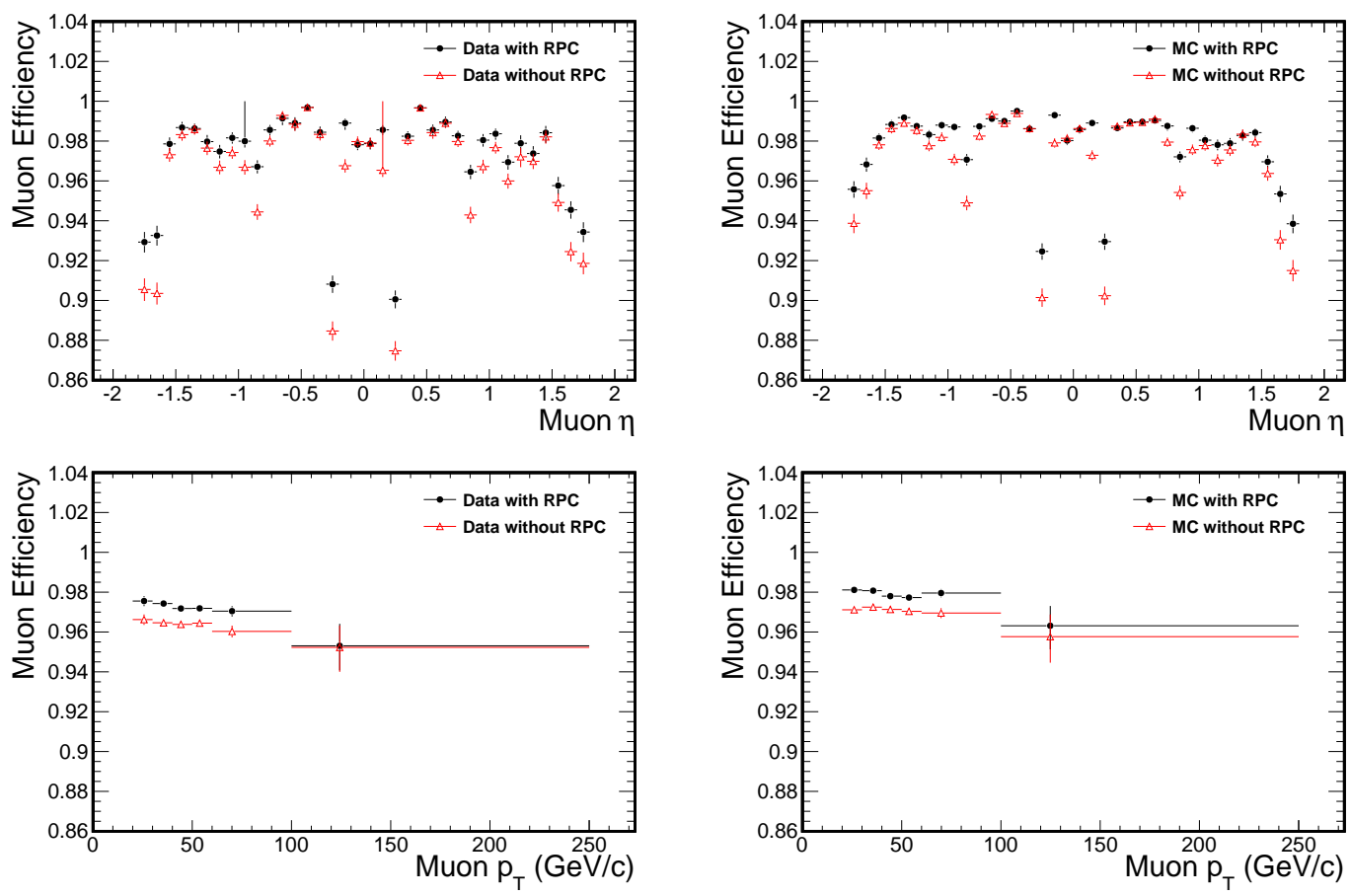

Figure 4: Efficiencies measured on data (left) and MC (right) for Loose muons as a function of $\eta$ (top) and $p_{T}$ (bottom) with (black dots) and without (red open triangle) the use of RPC in the track reconstruction.

are those that were matched by the global track reconstruction to a global muon reconstructed respectively with and without the use of RPC for the global-muon track fit, passing the selection.

For these efficiency measurements, a sample of events corresponding to an integrated luminosity of $\sim 1 \mathrm{fb}^{-1}$ has been used: the simulated process is $Z^{0} \rightarrow \mu^{+} \mu^{-}$only. Monte Carlo (MC) simulation of the background processes has not been included for the $Z^{0}$ decays, so that it would have been unfeasible for comparisons with the data, especially with the tight muon selection.

Figure 4 shows the reconstruction efficiencies for Loose muons given that a tracker track exists as measured from $Z^{0} \rightarrow \mu^{+} \mu^{-}$events collected in the $2011 \mathrm{LHC}$ run and the simulated events with high- $p_{T}$ single-muon triggers, not biasing the trigger measurement on the probe. 

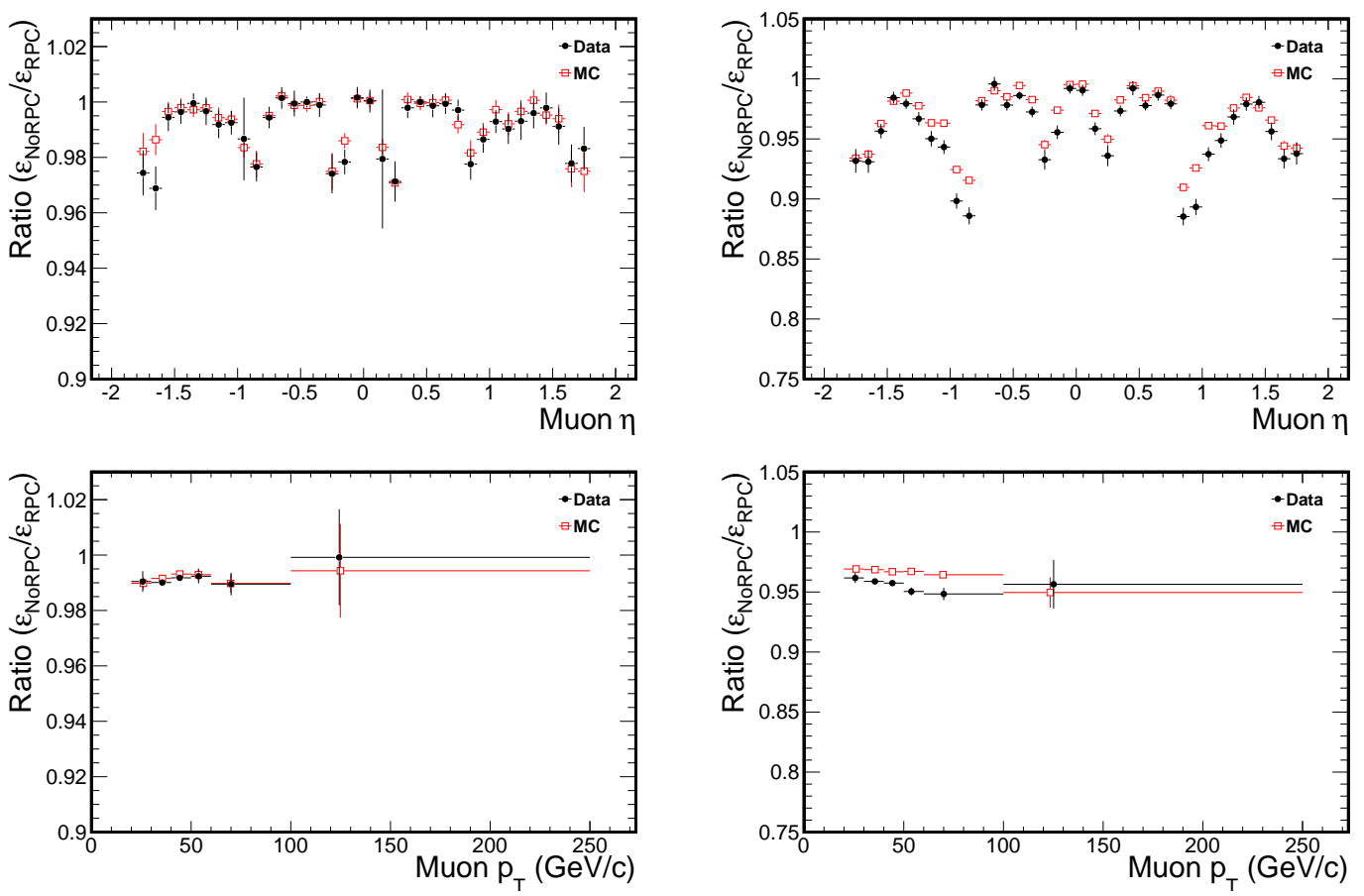

Figure 5: Ratio of efficiencies without RPC to with RPC for Loose (left) and Tight (right) muons as a function of $\eta$ (top) and $p_{T}$ (bottom). Results are shown for both data (black dots) and MC (red open square).

Figure 5 shows the results on the data with the ones extracted applying the same procedure on simulated events after the loose and tight muon selections, respectively. Exclusion of the RPC hits in the track fitting degrades the muon reconstruction efficiency by $1 \%(\sim 4 \%)$ on average, and to $3 \%(\sim 10 \%)$ in certain eta region for the loose (tight) muon selections.

\section{Conclusions}

The effect of the inclusion of the RPC measurements in the track fitting has been evaluated with $7 \mathrm{TeV}$ MC samples and 2011 collision data. The results show that, when the RPC hits are included in the global-muon track fit, there is an improvement in the efficiency by applying the muon selections with $p_{T}>20 \mathrm{GeV} / c$.

\section{Acknowledgments}

This work is supported by the Korean Research Foundation. We would like to thank Prof. Inkyu Park, University of Seoul, for his effective coordination of the Korea-CMS RPC project.

\section{References}

[1] CMS Collaboration, The CMS experiment at the CERN LHC, J. Instrum. 3 (2008) S08004.

[2] CMS Collaboration, Measurement of the charge ratio of atmospheric muons with the CMS detector, Phys. Lett. B 692 (2010) 83-104 [arXiv : 1005 . 5332]. 